\title{
Superexchange-Driven Magnetoelectricity in Magnetic Vortices
}

\author{
Kris T. Delaney, ${ }^{1}$ Maxim Mostovoy, ${ }^{2}$ and Nicola A. Spaldin ${ }^{3}$ \\ ${ }^{1}$ Materials Research Laboratory, University of California, Santa Barbara, California 93106-5121, USA \\ ${ }^{2}$ Zernike Institute for Advanced Materials, University of Groningen, The Netherlands \\ ${ }^{3}$ Materials Department, University of California, Santa Barbara, California 93106-5050, USA
}

(Dated: October 24, 2018)

\begin{abstract}
We demonstrate that magnetic vortices in which spins are coupled to polar lattice distortions via superexchange exhibit an unusually large linear magnetoelectric response. We show that the periodic arrays of vortices formed by frustrated spins on Kagomé lattices provide a realization of this concept; our $a b$ initio calculations for such a model structure yield a magnetoelectric coefficient that is 30 times larger than that of prototypical single phase magnetoelectrics. Finally, we identify the design rules required to obtain such a response in a practical material.
\end{abstract}

PACS numbers: $75.30 . \mathrm{Et}, 75.80 .+\mathrm{q}, 75.25 .+\mathrm{z}, 71.15 . \mathrm{Mb}$

The ability to control magnetism with electric fields, which can be realized through the interplay between spins and charges in solids, has an obvious technological appeal. The simplest form of such a control is the linear magnetoelectric effect, when an antiferromagnet placed in an electric field $\mathbf{E}$ becomes a magnetized with magnetization $\mathbf{M}$, while an applied magnetic field $\mathbf{H}$ induces an electric polarization $\mathbf{P}$, proportional to the field:

$$
\left\{\begin{aligned}
P_{i} & =\alpha_{i j} H_{j} \\
M_{j} & =\alpha_{i j} E_{i}
\end{aligned}\right.
$$

Here $\alpha_{i j}$ is the magnetoelectric tensor and summation over repeated indices is implied. This magnetoelectric response requires simultaneous breaking of inversion and time-reversal symmetries, which defines the allowed magnetic symmetry classes and the non-zero components of the magnetoelectric tensor. While the phenomenology of the linear magnetoelectric effect is now well understood [1, 2], the use of magnetoelectrics is hampered by rather low values of their magnetoelectric constants: for example in the prototypical magnetoelectric $\mathrm{Cr}_{2} \mathrm{O}_{3}$, a (large) electric field of $1 \times 10^{6} \mathrm{~V} / \mathrm{cm}$ induces a (tiny) magnetization of $\sim 9 \times 10^{-5} \mu_{B}$ per $\mathrm{Cr}$ ion. The search for materials with a much stronger response requires a deeper understanding of the microscopic mechanisms of magnetoelectric coupling and new ideas about spin orders and crystal lattices that can conspire to produce a large magnetoelectric effect.

The aforementioned technological driver is likely also responsible for the renewal of interest in the related class of multiferroic materials which have simultaneous ferroelectric and (ferro)magnetic orders. Two recent developments in this field are particularly relevant for the work we will present in this Letter. First, an early observation that spiral magnetic order can lead to an electrical polarization [3], has been confirmed repeatedly [4, 5] and the list of such materials has been considerably enlarged. While spectacular non-linear magnetoelectric effects, such as reorientation of electric polarization with a magnetic field, have been observed, the polarizations in such spiral magnets are small because the spin-lattice interaction is the weak spin-orbit-driven DzyaloshinskiiMoriya interaction [6, 7]. At the same time, a new class of multiferroics has been identified in which the magnetic ordering couples to the lattice through mechanisms of non-relativistic origin, in particular exchange striction arising from superexchange $[8,9]$. The stronger spinlattice coupling leads to correspondingly larger magnetically induced ferroelectric polarizations, with polarization values close to those of conventional ferroelectrics suggested.

In this Letter, we show that these two concepts from the field of multiferroics - symmetry breaking in spiral magnets, and super-exchange mediated spin-lattice coupling - can be combined to yield materials with strong linear magnetoelectric response.

We begin by considering the magnetoelectric response of a single spin vortex (Fig. 1a). This can be viewed as a magnetic spiral rolled into a circle, and so we can use the results from spiral multiferroics to analyze its magnetoelectric response. The magnetically induced ferroelectric polarization in spiral multiferroics is described by

$$
\mathbf{P} \propto \mathbf{e} \times \mathbf{Q},
$$

where $\mathbf{e}$ is the axis around which the spins rotate and $\mathbf{Q}$ is the spiral wavevector [10]. In our context, this coupling induces an inhomogeneous electric polarization locally oriented along the radial direction, so that the net polarization of the vortex is zero. A magnetic field applied in the $x y$ plane leads to a non-uniform rotation of spins in the vortex, which results in a nonzero net electric polarization proportional to the magnetic field (see Fig. 10). The spin vortex shown in Fig. 1h has a diagonal magnetoelectric tensor, with magnetization induced parallel to the applied electric field, while for the vortex shown in Fig. 1: an applied magnetic field induces a perpendicular electric polarization and the magnetoelectric tensor is antisymmetric (see Fig. 1 1 d). These conclusions are actually independent of the mechanism of magnetoelectric coupling and generally follow from the fact that 
the vortices shown in Figs. 17 and 1 have respectively a monopole moment $A \propto \sum_{\alpha} \mathbf{r}_{\alpha} \cdot \mathbf{S}_{\alpha}$ and a toroidal moment $\mathbf{T} \propto \sum_{\alpha} \mathbf{r}_{\alpha} \times \mathbf{S}_{\alpha} \quad[11]$.

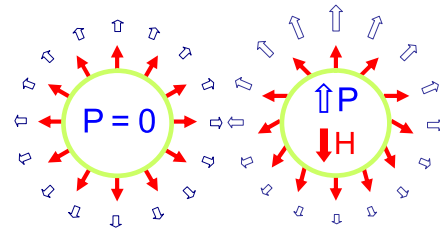

(a) (b)

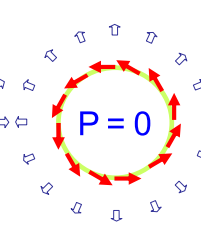

(c)

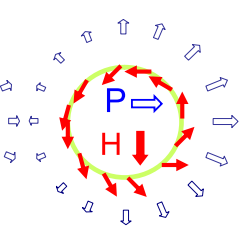

(d)
FIG. 1: (a) A magnetic vortex carrying a pseudoscalar moment. The thin solid arrows indicate the spin orientation, while the thick open arrows show the local polarization vector; (b) A magnetic field applied to the vortex shown in (a) induces a net polarization along the field direction; (c) A magnetic vortex carrying a toroidal moment; (d) A magnetic field applied to the vortex shown in (c) induces an electric polarization perpendicular to the field.

Next we analyze the spin-lattice coupling resulting from the dependence of the Heisenberg superexchange interaction between spins on their relative positions. Consider a three-atom unit consisting of two magnetic transition metal ions connected by a ligand such as oxygen that mediates superexchange (Fig. 22). Due to charge differences, the cations and ligand shift in opposite directions under application of an electric field. The exchange constant coupling the spins depends on the amplitude of the relative shifts through the changes in the metaloxygen distance and the metal-oxygen-metal bond angle $\theta$. According to the Anderson-Kanamori-Goodenough rules [12], the exchange is antiferromagnetic $(J>0)$ for $\theta=180^{\circ}$ and ferromagnetic $(J<0)$ for $\theta=90^{\circ}$. Experiments varying $\mathrm{A}$-site cation size in transition metal oxides have shown that the crossover from ferromagnetic to antiferromagnetic coupling is continuous [13]. Therefore, the total magnetization of the unit can be modified by applying an electric field; conversely changes in spin orientation will affect its electric dipole moment. (a)

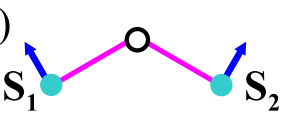

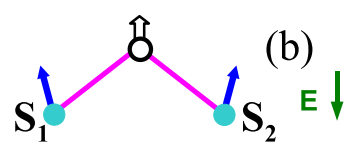

FIG. 2: (a) Two magnetic cations (solid circles) connected by a ligand (open circle); (b) Upon application of electric field the bond lengths and angle change resulting in a different relative alignment of spins $\mathbf{S}_{\mathbf{1}}$ and $\mathbf{S}_{\mathbf{2}}$.

Now we combine these two concepts to form a periodic array of magnetic vortices in which the magnetic moments are coupled through superexchange, and show that the combination leads to a large magnetoelectric response. The macroscopic magnetoelectric response of an array of magnetic vortices is proportional to the vortex density. Therefore we choose the smallest possible magnetic vortex as our building block: a triangle of antiferromagnetically coupled spins, in which the angle between spins in the lowest-energy state is $120^{\circ}$ (Fig. 3a). Using transition metal (TM) ions to provide the spins, and incorporating oxygen ligands between them (Fig. 3 a), leads to superexchange spin-spin interactions. Upon application of an electric field, the shifts of the oxygen anions relative to the positive TM ions induce changes in the Heisenberg exchange energy, changing the spin canting angles and resulting in a nonzero magnetization. The symmetry of the magnetoelectric response of the triangle is identical to that of the magnetic vortices of Fig. 1, with the form of the in-plane magnetoelectric tensor constrained by its $\mathrm{C}_{3 \mathrm{v}}$ symmetry to

$$
\alpha_{i j}=-\alpha_{0}\left(\begin{array}{cc}
\cos \varphi & \sin \varphi \\
-\sin \varphi & \cos \varphi
\end{array}\right)
$$

Here $\varphi$ is the angle between spins and the vectors directed from the triangle center to the corresponding vertex. In particular, $\varphi=0$ (Fig. 3h) leads to $\alpha_{i j}=-\alpha_{0} \delta_{i j}$, so that for $\alpha_{0}>0$ the induced magnetization $\mathbf{M}$ is antiparallel to the electric field $\mathbf{E}$; for $\varphi=\pi / 2, \mathbf{M}$ is perpendicular to $\mathbf{E}$ (Fig. 3b).
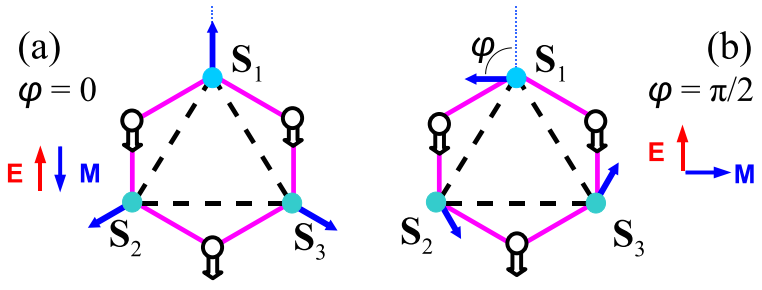

FIG. 3: Magnetoelectric response of a single TM-O triangular unit. For antiferromagnetic exchange coupling the angle between the spins $S_{1}, S_{2}$ and $S_{3}$ (solid arrows) in the zerofield ground state is $120^{\circ}$, so that the net magnetization is zero. For $\varphi=0$ [panel (a)] all spins are oriented out from the center of the triangle resulting in a nonzero pseudoscalar moment. Upon application of an electric field, $E$, the oxygen atoms (open circles) displace (open arrows) relative to manganese (solid circles) inducing a net magnetization through changes in the exchange coupling. The net magnetization $M$ is then opposite in direction to $E$ for $\varphi=0$, regardless of the orientation of $E$ with respect to the spins. For $\varphi=\frac{\pi}{2}$ [panel (b)] the spin triangle has a toroidal moment and the induced magnetization is perpendicular to the electric field.

To transform the concepts outlined above into a model material with a three-dimensional periodic structure, we begin with planes of $\mathrm{Mn}$ atoms situated on the vertices of a Kagomé lattice and assume that their spins form the $120^{\circ}$ structure with zero wave vector (see Fig. (4), as observed e.g. in iron jarosite $\mathrm{KFe}_{3}(\mathrm{OH})_{6}\left(\mathrm{SO}_{4}\right)_{2}$ [14]. At first glance, such a spin lattice would yield no magnetoelectric response because spins in the vortices formed 
at "up" and "down" triangles are oriented in opposite senses $(\varphi=0$ and $\varphi=\pi)$. However, when oxygen ions are positioned outside the "up" triangles and inside the "down" triangles, the sign of magnetoelectric coupling $\left[\alpha_{0}\right.$ in Eq. 3] also alternates and the contributions of all triangles to $\alpha_{i j}$ have the same sign. This can be understood by comparing the magnetoelectric response of the $\mathbf{S}_{2}-\mathbf{S}_{1}$ and $\mathbf{S}_{1}-\mathbf{S}_{2}^{\prime}$ spin pairs and noting that, for fixed bond lengths and angle, only the scalar product of spins is important in Heisenberg exchange.

The two-dimensional plane shown in Fig. प has a similar structure to the $\mathrm{MnO}$ layers of the experimentally realized $\mathrm{YMnO}_{3}$ structure 15], which consists of a connected mesh of oxygen trigonal bipyramids with $\mathrm{Mn}$ atoms at their centers. Using this structure as motivation, we extend our two-dimensional $\mathrm{MnO}$ planes to a three-dimensional periodic structure and introduce counter-ions ( $\mathrm{Ca}$ and $\mathrm{Al}$ ) in the voids of the lattice so that the correct charge balance is attained. To ensure that the sign of magnetoelectric response is the same for all layers, the neighboring $\mathrm{MnO}$ planes are rotated by $180^{\circ}$ with respect to each other (Fig. 5). This reverses the positioning of oxygen ions with respect to the "up" and "down" spin triangles in the next layer, which compensates the reversal of the spin direction that must result from the antiferromagnetic interlayer coupling provided by the $180^{\circ}$ connections through the apical oxygen atoms. Our resulting 'KITPite' structure 22], with chemical formula CaAlMn ${ }_{3} \mathrm{O}_{7}$, correctly breaks $I$ and $T$ symmetry; in addition the apical oxygen ions between the $\mathrm{MnO}$ layers are centers of combined IT symmetry.

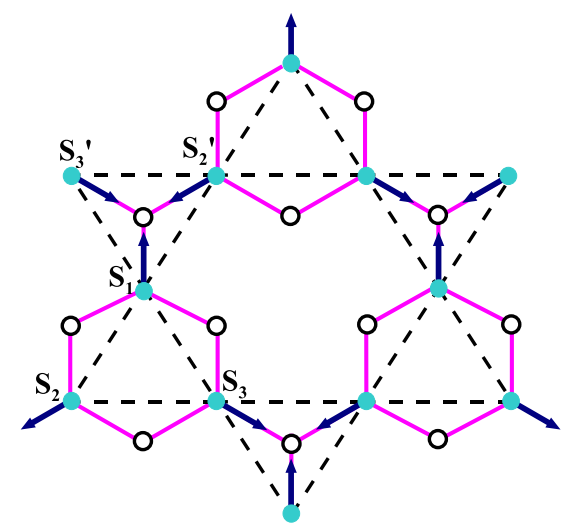

FIG. 4: The structure of one $\mathrm{MnO}$ plane. The Mn atoms (solid circles) are arranged on a Kagomé lattice (dashed lines) with oxygen atoms (open circles) mediating the binding and superexchange.

Finally, to assess the strength of the magnetoelectric response of the model material introduced in the previous section, we turn to first principles calculations employing plane-wave density functional theory (DFT) as implemented in VASP [16]. We use PAW potentials for core-

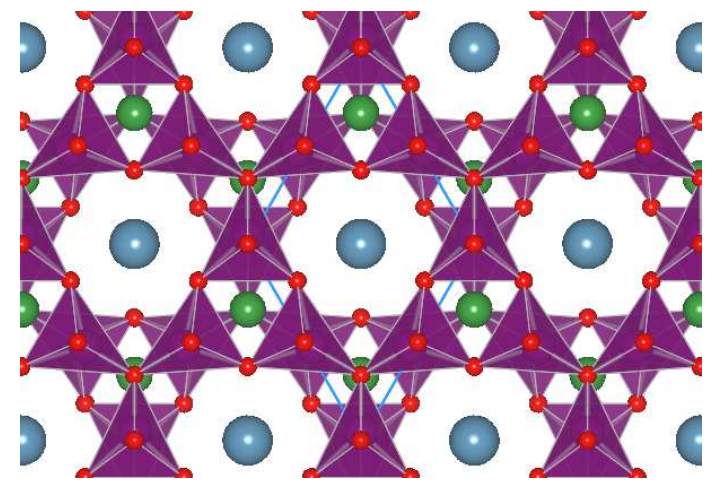

FIG. 5: Two layers of KITPite in the relaxed structure (space group Pmma). The Mn ions are at the center of the oxygen trigonal bipyramids (purple polyhedra). The correct charge balance is obtained through $\mathrm{Ca}$ (blue) and $\mathrm{Al}$ (green) counter ions situated in voids of the $\mathrm{MnO}$ mesh.

valence separation [17], and include non-collinear magnetism for the valence electrons. We approximate the exchange-correlation part of the Kohn-Sham potential using the rotationally invariant form of the LSDA $+U$ in the fully localized limit[18], with the Hubbard $U$ applied only to the $\mathrm{Mn} d$ electrons $(U=5.5 \mathrm{eV}$ and $J=0.5 \mathrm{eV}[19])$. It is important to note that we deliberately do not include spin-orbit coupling in our calculations so as to ensure that our calculated magnetoelectric response arises entirely from the superexchange coupling. As a result, the pseudoscalar and toroidal spin arrangements (Figs. 3 (a) and (b)) are degenerate, and we are unable to determine whether $\alpha$ is diagonal or antisymmetric.

We first relax the structure in the absence of an electric field by optimizing the ionic coordinates to find the lowest-energy state with the constraint that the trigonal bipyramids are prevented from tilting. This constraint preserves the Kagomé structure of the $\mathrm{MnO}$ planes which is essential for demonstrating the superexchange magnetoelectricity of this model. The resulting structure is shown in Fig. 5. Our calculated valence electronic structure is, as expected, similar to that of $\mathrm{YMnO}_{3}$ 15]: The formal Mn charge is $3+$, with four majority $d$ electrons per Mn $\left(d_{x y}, d_{x^{2}-y^{2}}, d_{y z}\right.$ and $\left.d_{x z}\right)$, and an unoccupied minority channel providing a local moment of $4 \mu_{\mathrm{B}} / \mathrm{Mn}$.

Subsequently, we apply an electric field and calculate the linear response of the ions, which is sufficient for computing the linear magnetoelectric coefficient. The force on an ion upon application of an external electric field is determined by the Born effective charge tensor, $Z^{\star}$, through $F_{\mu i}=Z_{\mu i j}^{\star} E_{j}$, where $\mathbf{F}$ is the force, $\mathbf{E}$ is the applied electric field, $\mu$ is an index denoting the ion, and $i, j$ are spatial directions. The summation convention for repeated indices is once again employed. All elements of the $Z^{\star}$ tensor are computed through derivatives of the 
bulk polarization $Z_{\mu i j}^{\star}=\frac{\delta P_{j}}{\delta R_{\mu i}}$, where $\mathbf{P}$ is calculated using the Berry-phase approach [20] for a small displacement in all degrees of freedom individually. The method is close to that employed by Íniguez [21].

In order to obtain the first-order ionic response to the field, we use the force-constant matrix $C_{\mu i, \nu j}=\frac{\delta F_{\mu i}}{\delta R_{\nu j}}$. Then, to linear order, the ionic displacements for a given force are found by inverting the force-constant matrix through $\delta R_{\nu j}=C_{\nu j, \mu i}^{-1} \delta F_{\mu i}$, so that the ionic response to an applied electric field is $\delta R_{\nu j}=C_{\nu j, \mu i}^{-1} Z_{\mu i k}^{\star} E_{k}$. Finally, the total magnetization is calculated as a function of $E_{k}$, yielding the linear magnetoelectric coupling constant.

Fig. 6] shows the calculated magnitude of the induced magnetization as a function of applied electric field. With a field of $1 \times 10^{6} \mathrm{~V} / \mathrm{cm}$, the ionic response leads to an average displacement of $\mathrm{Mn}$ atoms of $0.007 \AA$ in the direction of the field and of $\mathrm{O}$ atoms of $0.005 \AA$ against the field. The Born effective charges, $Z^{\star}$, have an in-plane average magnitude of $+3.30 e^{-}$for $\mathrm{Mn}$ and $-2.26 e^{-}$for O. Using an equilibrium spin arrangement as shown in Fig. 4, the spins $S_{2}$ and $S_{3}$ rotate by $-0.1^{\circ}$ and $0.1^{\circ}$ respectively, leading to a magnetoelectric coupling coefficient of $\alpha=1.10 \times 10^{-5} \mathrm{JT}^{-1} \mathrm{~V}^{-1} \mathrm{~m}^{-2}$. Transformation to regularized CGS units yields $\alpha_{\mathrm{CGS}}=4.15 \times 10^{-3}$. For a benchmark, we compare to the magnetoelectric response of $\mathrm{Cr}_{2} \mathrm{O}_{3}$ computed also within density functional theory 21], $\alpha\left(\mathrm{Cr}_{2} \mathrm{O}_{3}\right)=1.3 \times 10^{-4}$ in Gaussian units (in good agreement with the experimental value). Hence, our model system has a magnetoelectric coupling around 30 times larger than that of $\mathrm{Cr}_{2} \mathrm{O}_{3}$. Since the spin-orbit coupling was not considered in this work, magnetic anisotropies that determine the angle $\varphi$ were neglected. We therefore cannot predict whether KITPite would carry a pseudoscalar or a toroidal moment in the ground state. However, the strength of the magnetoelectric coupling resulting from Heisenberg superexchange $\left[\alpha_{0}\right.$ in Eq.(3)] is insensitive to $\varphi$.

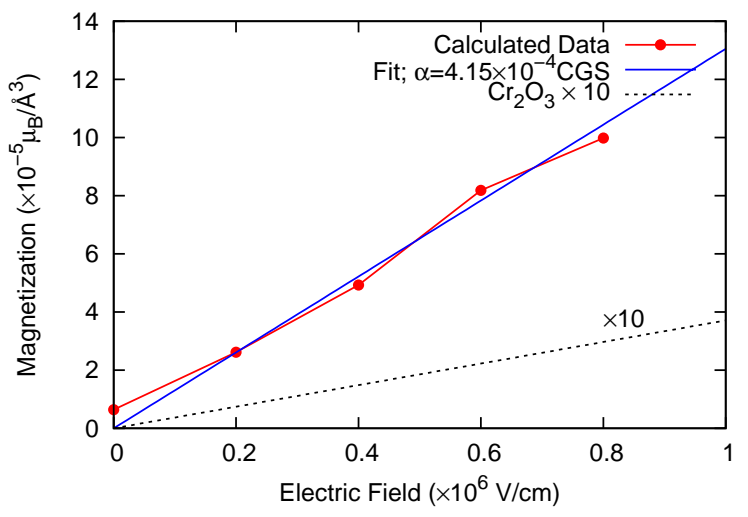

FIG. 6: Calculated magnetoelectric response of the model system using density functional theory and a linear fit.
In conclusion, we have combined the concepts of magnetically induced polarization in magnetic vortices with lattice-mediated coupling through the superexchange mechanism to demonstrate strong magnetoelectric coupling in geometrically frustrated antiferromagnets. We showed that such a mechanism can be studied using modern density-functional theory approaches with noncollinear spin density functionals and augmented with linear-response methods, and we explicitly calculated the magnetoelectric coupling of a model transition metal oxide. While the linear magnetoelectric response of our model compound is larger than that of any known single phase material, we anticipate that many further improvements are possible: In particular materials with larger polarizability through increased $Z^{\star} \mathrm{s}$ or reduced rigidity would be promising. We hope that this study will stimulate the search for additional novel strongly-coupled magnetoelectric materials.

This work was initiated during the research program on Moments and Multiplets in Mott Materials at the Kavli Institute for Theoretical Physics at UC Santa Barbara under the NSF grant No. PHY05-51164. Delaney and Spaldin were supported by the National Science Foundation under Award No. DMR-0605852. Calculations were performed at the UCSB California Nanosystems Institute (CNSI) with facilities provided by NSF Award No. CHE-0321368 and Hewlett-Packard, at the San Diego Supercomputer Center, and at the National Center for Supercomputer Applications.

[1] L. D. Landau and E. M. Lifshitz, Electrodynamics of Continuous Media (Addison-Wesley, Reading, MA, USA, 1960).

[2] M. Fiebig, J. Phys. D: Appl. Phys. 38, R123 (2005).

[3] R. E. Newnham, J. J. Kramer, W. A. Schulze, and L. E. Cross, J. Appl. Phys. 49, 12 (1978).

[4] T. Kimura, T. Goto, H. Shintani, K. Ishizaka, T. Arima, and Y. Tokura, Nature 426, 55 (2003).

[5] T. Goto, T. Kimura, G. Lawes, A. P. Ramirez, and Y. Tokura, Phys. Rev. Lett. 92, 257201 (2004).

[6] H. Katsura, N. Nagaosa, and A. V. Balatsky, Phys. Rev. Lett. 95, 057205 (2005).

[7] I. A. Sergienko and E. Dagotto, Phys. Rev. B 73, 094434 (2006).

[8] I. A. Sergienko, C. Sen, and E. Dagotto, Phys. Rev. Lett. 97, 227204 (2006).

[9] S. Picozzi, K. Yamaguchi, B. Sanyal, I. A. Sergienko, and E. Dagotto, Phys. Rev. Lett. 99, 227201 (2007).

[10] M. Mostovoy, Phys. Rev. Lett. 96, 067601 (2006).

[11] N. A. Spaldin, M. Fiebig, and M. Mostovoy, J. Phys.: Condens. Matter in press (????).

[12] P. W. Anderson, Magnetism, vol. 1 (Academic Press, New York, 1963).

[13] M. A. Subramanian, A. P. Ramirez, and W. J. Marshall, Phys. Rev. Lett. 82, 1558 (1999).

[14] D. Grohol, K. Matan, J.-H. Cho, S.-H. Lee, J. W. Lynn, 
D. G. Nocera, and Y. S. Lee, Nature Materials 4, 323 (2005).

[15] B. B. Van Aken, T. T. M. Palstra, A. Filippetti, and N. A. Spaldin, Nature Materials 3, 164 (2004).

[16] G. Kresse and J. Furthmüller, Phys. Rev. B 54, 11169 (1996).

[17] G. Kresse and D. Joubert, Phys. Rev. B 59, 1758 (1999).

[18] A. I. Liechtenstein, V. I. Anisimov, and J. Zaanen, Phys. Rev. B 52, R5467 (1995).
[19] Z. Yang, Z. Huang, L. Ye, and X. Xie, Phys. Rev. B 60, 15674 (1999).

[20] R. D. King-Smith and D. Vanderbilt, Phys. Rev. B 47, 1651 (1993).

[21] J. Íñiguez, Phys. Rev. Lett. 101, 117201 (2008).

[22] After the Kavli Institute for Theoretical Physics, where this structure was first suggested by the authors. 